\title{
Live Color SEM Imaging
}

Ernst Jan Vesseur ${ }^{1}$

1. Thermo Fisher Scientific, Eindhoven, the Netherlands.

* Corresponding author: ernst.jan.vesseur@thermofisher.com

The human observer typically has a preference for color images over black and white (greyscale). As a result, most imaging techniques are in color and all modern media and display technologies have transitioned to full color output. This has led the users of scanning electron microscopy, a greyscale technique, to regularly add color to their images using artificial methods, even when these colors do not carry a scientific meaning.

Techniques such as EDS, EBSD, CL and others are able to provide SEM images with meaningful color, but they are usually not integrated with imaging and navigation, they often require separate softwares which makes them difficult to use, and they are inherently slow.

Here, we present live color SEM imaging in a commercially available SEM platform. Combining the signals from secondary electrons, backscatter electrons and x-rays in one integrated scanning system, images are colored live based on image features and elemental composition (example in Figure 1). The direct display of color images provides an intuitive way of working with a specimen, it provides the shortest time to analytical result, and it is easier to interpret. We demonstrate that live color imaging is a completely new way of collecting EDS information in a system that is always on, and that has no barriers to anyone working with the microscope, while providing images with a color that is actually meaningful. 


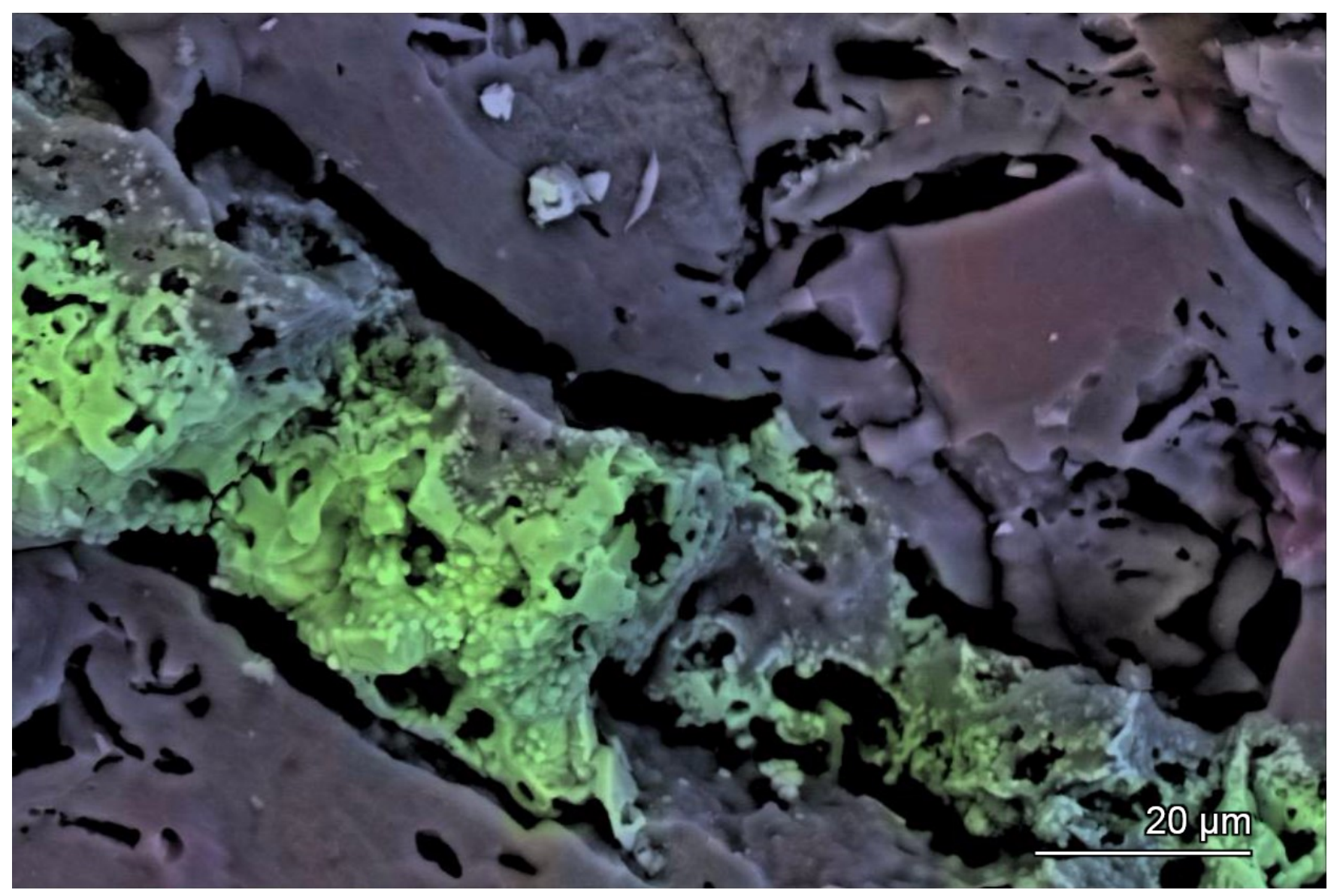

Figure 1. Ceramic material with an iron inclusion (green). 